\title{
Long-Term Persistence of Serum-Specific Anti-Chikungunya IgM Antibody - A Case Series of Brazilian Patients
}

\author{
Denise Maria do Nascimento Costa ${ }^{[1],}$ Maria Rosângela Cunha Duarte Coêlhoo ${ }^{[2],[3],}$ \\ Pedro Alves da Cruz Gouveia ${ }^{[1]}$, Luan Araújo Bezerra ${ }^{[2]}$, Claudia Diniz Lopes Marques ${ }^{[1]}$, \\ Angela Luzia Branco Pinto Duarte ${ }^{[1]}$, Lucila Maria Valente ${ }^{[1]}$ and Vera Magalhães ${ }^{[1]}$
}

\author{
[1]. Universidade Federal de Pernambuco, Hospital das Clínicas, Recife, PE, Brasil. \\ [2]. Universidade Federal de Pernambuco, Departamento de Virologia, Laboratório de Imunopatologia Keizo Asami, Recife, PE, Brasil. \\ [3]. Universidade Federal de Pernambuco, Centro de Biociências, Departamento de Fisiologia e Farmacologia, Recife, PE, Brasil.
}

\begin{abstract}
The persistence of serum-specific anti-chikungunya IgM antibodies (CHIKV-IgM) can vary after chikungunya fever (CHIK) infection. However, the factors related to its production are not yet known. We described a case series drawn up from data collected from 57 patients between 12 and 36 months after the acute phase of CHIK infection in Northeastern Brazil. CHIKV-IgM was detectable in 7/57 $(12.3 \%)$ patients after 28.3 months of infection. No frequency differences in chronic musculoskeletal manifestations and underlying conditions were detected between patients with or without CHIKV-IgM. CHIKV-IgM was detected for up to 35 months in Brazilian patients after CHIK infection.
\end{abstract}

Keywords: Chikungunya fever. Immunoglobulin M. Arthritis.

\section{INTRODUCTION}

Chikungunya virus (CHIKV) is an alphavirus of the Togaviridae family, transmitted by Aedes mosquitoes and causes chikungunya fever $(\mathrm{CHIK})^{1}$. Although it was first described in 1952, CHIKV was first observed in Brazil in 2014, and during the subsequent epidemic, more than 400.000 cases were reported ${ }^{1,2}$. In Northeastern Brazil, the state of Pernambuco recorded a higher number of deaths and an incidence rate almost four times higher than the rest of the country ${ }^{2}$. This disparity has raised questions regarding the course of CHIK in our intensely admixed population ${ }^{3}$.

It is known that in the viremia phase, CHIKV causes B-cell activation and early production of immunoglobulins, with the detection of serum-specific anti-chikungunya IgM antibodies (CHIKV-IgM) expected from the onset of infection until approximately three months after infection ${ }^{4}$. Studies conducted in different regions of the world have demonstrated that the persistence

\footnotetext{
Corresponding author: Denise Maria do Nascimento Costa.

e-mail: denisecosta_81@yahoo.com

(iD) https://orcid.org/0000-0001-7306-7044

Received 6 January 2021

Accepted 26 February 2021
}

of CHIKV-IgM may be quite variable. However, most studies have been limited to a period of up to 18 months after infection, and none have included Brazilian patients ${ }^{4-10}$.

Although this antibody does not present an established pathogenic mechanism, it has been questioned whether there is a relationship between the serum persistence of this immunoglobulin and chronic joint symptoms in CHIK ${ }^{11,12}$. Notably, the reason that some patients develop chronic arthralgia is poorly understood. Chronic forms of CHIK occur in approximately $10-60 \%$ of patients and may cause persistent, severe disability, which significantly decreases quality of $\operatorname{life}^{1}$. Some authors have controversially suggested that the chronicity of CHIK symptoms may be related to the presence of pre-existent comorbidities ${ }^{13,14}$. Thus, this case series aimed to identify the frequency of detectable serum CHIKV-IgM in patients with chronic symptoms from 12 to 36 months after CHIKV infection in a Brazilian university hospital, describing the chronic musculoskeletal manifestations and previous comorbidities of those with positive antibodies.

\section{CASE REPORT}

From December 2018 to July 2019, a case series was prepared from data collected from 57 patients diagnosed with CHIK, who were previously evaluated in a cross-sectional study, between 12 
and 36 months after the acute phase of infection. These patients were followed-up in a specific outpatient clinic for people with chronic symptoms after CHIK infection at the Hospital das Clínicas at the Universidade Federal de Pernambuco, Northeastern Brazil. A diagnosis of CHIK was established based on clinical and epidemiological criteria and confirmed by the detection of serum CHIKV-IgM or IgG by enzyme-linked immunosorbent assay (ELISA). The study was approved by the local ethics committee, and consent forms were obtained from all patients before inclusion in the study.

At the time of the evaluation, blood samples were collected and tested for CHIKV-IgM using a commercially available qualitative ELISA, following the manufacturer's instructions (anti-chikungunya virus IIFT; Euroimmun, Germany). All patients underwent clinical examinations by experienced rheumatologists. We compared the frequency of chronic musculoskeletal manifestations and comorbidities in the two groups of patients: CHIKV-IgM-positive and CHIKV-IgM-negative. Data were analyzed using SPSS software and Fisher's exact test. The significance level was set at $\mathrm{p}<0.05$.
Between 12 and 36 months after CHIK acute infection, seven of 57 patients (12.3\%) presented detectable CHIKV-IgM with a median time of 28.3 months (range, 15-35 months). Six of seven patients (86\%) who presented with positive CHIKV-IgM reported persistent musculoskeletal symptoms. All these patients displayed joint pain, and two experienced persistent arthritis. The descriptive characteristics of the patients with persistent detectable CHIKV-IgM are shown in Table 1.

Patients positive for CHIKV-IgM were compared to those without detectable antibodies with respect to the presence of chronic musculoskeletal manifestations and underlying previous disease. The descriptive characteristics of the patients are presented in Table 2. Most of the 57 patients were female $(86 \%)$, with a mean age of 52.2 years (SD, 11.4). The mean period since CHIK diagnosis was 31.8 months (SD, 5.7). Approximately $53 \%$ of the patients presented with underlying conditions, mainly hypertension and diabetes. In this sample, no differences were detected between the groups with positive or negative CHIKV-IgM in terms of the frequency of patients with persistent chronic musculoskeletal symptoms $(86 \% \times 82 \%, \mathrm{p}=0.76)$ or the presence of previous underlying chronic conditions $(71 \% \times 50 \%, \mathrm{p}=0.25)$ (Table 2$)$.

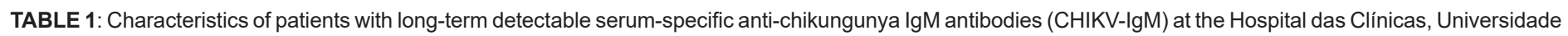
Federal de Pernambuco, Brazil.

\begin{tabular}{|c|c|c|c|c|c|}
\hline Patients & Demographics $^{1}$ & Comorbidities $^{2}$ & $\begin{array}{l}\text { Symptoms in acute } \\
\text { phase }\end{array}$ & Persistent joint pain & $\begin{array}{l}\text { Time since acute } \\
\text { infection (months) }\end{array}$ \\
\hline 1 & 63y, M, White & MG, HTN & Fever/joint pain & Yes & 15 \\
\hline 2 & 50y, F, Nonwhite & HTN, DM & Fever/joint pain/rash & No & 23 \\
\hline 3 & $60 y, F$, Nonwhite & HTN, DM & Fever/joint pain & Yes & 24 \\
\hline 4 & $69 y, F$, Nonwhite & HTN & Joint pain/rash & Yes & 32 \\
\hline 5 & 51y, F,Nonwhite & - & Fever/joint pain/rash & Yes & 32 \\
\hline 6 & 44y, F, Nonwhite & HTN & Fever/joint pain/rash & Yes & 34 \\
\hline 7 & 39y, F, White & - & Fever/joint pain & Yes & 35 \\
\hline
\end{tabular}

${ }^{1}$ Age (years); Sex (M=male, F=female), race; ${ }^{2 M G}$ : membranous glomerulopathy; HTN: hypertension; DM: diabetes mellitus.

TABLE 2: Characteristics of 57 patients with persistent Chikungunya fever (CHIK) for more than 12 months, monitored at the Hospital das Clínicas, Universidade Federal de Pernambuco, Brazil.

\begin{tabular}{|c|c|c|c|}
\hline Characteristics $^{1}$ & $\begin{array}{l}\text { All patients } \\
(N=57)\end{array}$ & $\begin{array}{l}\text { CHIKV-IgM positive } \\
\qquad(\mathrm{N}=7)\end{array}$ & $\begin{array}{l}\text { CHIKV-IgM negative } \\
\qquad(\mathrm{N}=50)\end{array}$ \\
\hline Age, years ${ }^{2}$ & $52.2(11.4)$ & $53.7(10.7)$ & $52.0(11.6)$ \\
\hline \multicolumn{4}{|l|}{ Sex } \\
\hline Female, N (\%) & $49(86 \%)$ & $6(85.7 \%)$ & $43(86.0 \%)$ \\
\hline Male, N (\%) & $8(14 \%)$ & $1(14.3 \%)$ & $7(14.0 \%)$ \\
\hline \multicolumn{4}{|l|}{ Race } \\
\hline White, N (\%) & $17(29.8 \%)$ & $1(14.3 \%)$ & $16(32.0 \%)$ \\
\hline Nonwhite, $\mathrm{N}(\%)$ & $40(70.2 \%)$ & $6(86.7 \%)$ & $34(68.0 \%)$ \\
\hline Time (in months) of CHIKV-IgM assessment after CHIK acute phase ${ }^{2}$ & $31.8(5.7)$ & $28.3(7.7)$ & $32.3(5.3)$ \\
\hline Comorbidities, N (\%) & $30(52.6 \%)$ & $5(71.4 \%)$ & $25(50.0 \%)$ \\
\hline Hypertension, $\mathrm{N}(\%)$ & $24(42.1 \%)$ & $5(71.4 \%)$ & $19(38.0 \%)$ \\
\hline Diabetes mellitus, $\mathrm{N}(\%)$ & $10(17.5 \%)$ & $2(28.6 \%)$ & $8(16.0 \%)$ \\
\hline Hypothyroidism, N (\%) & $5(8.8 \%)$ & $0(0.0 \%)$ & $5(10.0 \%)$ \\
\hline Persistent chronic musculoskeletal symptoms, N (\%) & $47(82.4 \%)$ & $6(85.7 \%)$ & $41(82.0 \%)$ \\
\hline
\end{tabular}

${ }^{1} \mathrm{p}$-value $>0.05$ was established for all characteristics; ${ }^{2}$ mean (SD). CHIK: chikungunya fever; CHIKV-IgM: serum-specific anti-chikungunya IgM antibodies. 
TABLE 3: Persistence of serum-specific IgM-chikungunya antibodies (CHIKV-IgM) in studies according to the time elapsed after chikungunya fever and the country of evaluation.

\begin{tabular}{lccc}
\hline Author, year & Country & Time (months) & CHIKV-IgM+ patients/total (\%) \\
\hline Grivard et al., 2007 [4] & Ilhas Reunion & $13-18$ & $25 / 45(55.5)$ \\
Chopra et al., 2008 [10] & India & $3-6$ & $19 / 30(63)$ \\
Oliver et al., 2009 [5] & France & 13 & $2 / 18(11.1)$ \\
Demanou et al., 2010 [6] & Cameroon & 12 & $54 / 105(51.4)$ \\
Moro et al., 2012 [7] & Italy & $12-13$ & $30 / 227(13.2)$ \\
Pierro et al., 2015 [8] & Italy & $12-13$ & $23 / 133(17.3)$ \\
Chelluboina et al., 2019 [9] & India & 12 & $4 / 21(19)$ \\
\hline
\end{tabular}

${ }^{1}$ time elapsed after chikungunya fever; CHIKV-IgM: serum-specific anti-chikungunya IgM antibodies.

\section{DISCUSSION}

CHIK was first observed in Brazil in the Northern and Northeastern regions. Northeastern Brazil was severely affected by the epidemic, and it was necessary to establish a specific outpatient clinic for CHIK at the Hospital das Clínicas at the Universidade Federal de Pernambuco due to the high frequency of chronic manifestations. This led us to assess whether the long-term progression of CHIK in Brazilian patients was different from that in patients from other affected countries. Herein, we present the serological and clinical data of patients with long-term detectable CHIKV-IgM followed in a specific outpatient clinic for people with chronic symptoms after CHIK in Northeastern Brazil.

According to initial studies, after CHIKV infection, there is a progressive reduction in the production of CHIKV-IgM, which was detected in $76.2 \%$ of patients between two and six months, $69.8 \%$ between seven and 12 months, and $55.5 \%$ between 13 and 18 months after the acute phase ${ }^{4}$. Subsequent studies carried out in different regions of the world have demonstrated that CHIKV-IgM production may vary according to the time lapse between acute infection and the assessed population (Table 3). In Africa, CHIKVIgM could be detected in up to $51 \%$ of patients approximately one year after CHIK, while Italy reported lower percentages of detectable antibodies, ranging from $13 \%$ to $17 \%$, after a similar period $^{6-8}$. In this study, we observed a frequency of CHIKV-IgM positivity comparable to that in Italian studies (12\%), although with assessments performed at different periods.

It is plausible to accept that the difference observed in the present study may only be related to the time elapsed since the acute phase of CHIK as our assessment occurred after a mean interval of 28 months, which is longer than the intervals in previous studies. Furthermore, a commercially available ELISA kit was used in the present study; this may have reduced the sensitivity for detecting such antibodies ${ }^{1}$. We may also speculate that the long-term production of CHIKV-IgM could be related to the CHIKV genotype, host genetic factors, or epigenetic factors related to the environment, in addition to possible continuous antigenic stimulation.

Whether the persistence of CHIKV-IgM in the chronic phase of CHIK is related to a possible pathogenic role has not yet been fully established. Studies that only assessed individuals with persistent joint pain in the chronic phase of CHIK have demonstrated detectable CHIKV-IgM in around 63\% after six months and in $11-19 \%$ after one year ${ }^{5,9,10}$. CHIKV-IgM was also detected in patients who had destructive arthritis up to three years after CHIKV infection, corroborating the hypothesis that it is persistently produced due to the maintenance of a tissue reservoir in the host $\mathrm{t}^{10,12,15}$. Although our small sample size does not allow definitive conclusions, our results are similar to those of other studies that did not report a correlation between the presence of CHIKV-IgM and chronic joint manifestations of CHIK. For example, Moro and Borgherini detected CHIKV-IgM in $40 \%$ of patients up to 18.7 months after infection, with no association with persistent arthralgia ${ }^{7,11}$.

In addition, some studies have controversially reported that the severity of CHIK, as well as the chronicity of symptoms, may be related to the presence of comorbidities, such as hypertension and diabetes ${ }^{13,14}$. However, no studies have assessed whether the presence of these comorbidities affects the duration of IgM production. In our study, the frequency of underlying previous conditions did not differ between patients with positive and negative CHIKV-IgM, which may suggest that the presence of comorbidities is not related to the persistent production of these antibodies in the long-term.

In summary, in contrast to most studies that have analyzed patients up to $12-18$ months post-CHIKV infection, this case series detected CHIKV-IgM for up to 35 months in $12.3 \%$ of Brazilian patients. The presence of persistent joint symptoms and underlying conditions did not seem to be associated with the longterm production of CHIKV-IgM in this sample, although further investigations are required to validate these observations. This study has indicated that a positive serological test for CHIK-IgM alone should not define the diagnosis of a recent disease.

\section{ACKNOWLEDGMENTS}

We thanks to the institution that provided technical support for the development of this study.

\section{AUTHORS' CONTRIBUTION}

DMNC: Conception and design of the study, Acquisition of data, Drafting the article; MRCDC: Conception and design of the study, Acquisition of data; Final approval of the version to be submitted; PACG: Analysis and interpretation of data, Drafting the article; 
LAB: Acquisition of data; Analysis and interpretation of data; CDLM: Conception and design of the study, Acquisition of data; Final approval of the version to be submitted; ALBPD: Conception and design of the study, Acquisition of data; Final approval of the version to be submitted; LMV: Conception and design of the study, Acquisition of data; Final approval of the version to be submitted; VMS: Conception and design of the study, Acquisition of data; Final approval of the version to be submitted;

\section{CONFLICT OF INTEREST}

The authors declare that there is no conflict of interest.

\section{FINANCIAL SUPPORT}

None.

\section{ORCID}

Denise Maria do Nascimento Costa:0000-0001-7306-7044

Maria Rosângela Cunha Duarte Coêlho:0000-0002-5671-5756

Pedro Alves da Cruz Gouveia:0000-0002-7708-5132

Luan Araújo Bezerra:0000-0002-1502-8730

Claudia Diniz Lopes Marques:0000-0002-3333-2621

Angela Luzia Branco Pinto Duarte:0000-0001-6434-9939

Lucila Maria Valente:0000-0003-2908-4212

Vera Magalhães da Silveira:0000-0003-3495-0234

\section{REFERENCES}

1. Rougeron V, Sam IC, Caron M, Nkoghe D, Leroy E, Roques P. Chikungunya, a paradigm of neglected tropical disease that emerged to be a new health global risk. J Clin Virol. 2015;64:144-52.

2. Ministério da Saúde (MS). Secretaria de Vigilância Epidemiológica. Monitoramento dos casos de dengue, febre de chikungunya e febre pelo vírus Zika até a Semana Epidemiológica 52, 2015. Bol Epidemiológico. 2016;47(3):1-10.

3. Coelho AV, Moura RR, Cavalcanti CA, Guimarães RL, Sandrin-Garcia $\mathrm{P}$, Crovella $\mathrm{S}$, et al. A rapid screening of ancestry for genetic association studies in an admixed population from Pernambuco, Brazil. Genet Mol Res. 2015;14(1):2876-84.
4. Grivard P, Le Roux K, Laurent P, Fianu A, Perrau J, Gigan J, et al. Molecular and serological diagnosis of Chikungunya virus infection. Pathol Biol. 2007;55(10):490-4.

5. Oliver M, Grandadam M, Marimoutou C, Rogier C, Botelho-Nevers E, Tolou $\mathrm{H}$, et al. Persisting mixed cryoglobulinemia in Chikungunya infection. PLoS Negl Trop Dis. 2009;3(2):1-10.

6. Demanou M, Antonio-Nkondjio C, Ngapana E, Rousset D, Paupy C, Manuguerra JC, et al. Chikungunya outbreak in a rural area of Western Cameroon in 2006: A retrospective serological and entomological survey. BMC Res Notes. 2010;3(1):128.

7. Moro ML, Grilli E, Corvetta A, Silvi G, Angelini R, Mascella F A, et al. Long-term chikungunya infection clinical manifestations after an outbreak in Italy: A prognostic cohort study. J Infect. 2012;65(2): $165-72$.

8. Pierro A, Rossini G, Gaibani P, Finarelli AC, Moro ML, Landini MP, et al. Persistence of anti-chikungunya virus-specific antibodies in a cohort of patients followed from the acute phase of infection after the 2007 outbreak in Italy. New microbes new Infect. 2015;7:23-5.

9. Chelluboina S, Robin S, Aswathyraj S, Arunkumar G. Persistence of antibody response in chikungunya. VirusDisease. 2019;30(3):469-73.

10. Chopra A, Anuradha V, Lagoo-Joshi V, Kunjir V, Salvi S, Saluja M. Chikungunya virus aches and pains: An emerging challenge. Arthritis Rheum. 2008;58(9):2921-2.

11. Borgherini G, Poubeau P, Jossaume A, Gouix A, Cotte L, Michault A, et al. Persistent Arthralgia Associated with Chikungunya Virus: A Study of 88 Adult Patients on Reunion Island. Clin Infect Dis. 2008;47(4): 469-75.

12. Malvy D, Ezzedine K, Mamani-Matsuda M, Autran B, Tolou H, Receveur MC, et al. Destructive arthritis in a patient with chikungunya virus infection with persistent specific IgM antibodies. BMC Infect Dis. 2009;9(1):200.

13. Zaid A, Gérardin P, Taylor A, Mostafavi H, Malvy D, Mahalingam S. Chikungunya Arthritis: Implications of Acute and Chronic Inflammation Mechanisms on Disease Management. Arthritis Rheumatol. 2018;70(4):484-95.

14. Economopoulou A, Dominguez M, Helynck B, Sissoko D, Wichmann O, Quenel P, et al. Atypical Chikungunya virus infections: Clinical manifestations, mortality and risk factors for severe disease during the 2005-2006 outbreak on Réunion. Epidemiol Infect. 2009;137(4):534-41.

15. Labadie K, Larcher T, Joubert C, Mannioui A, Delache B, Brochard P, et al. Chikungunya disease in nonhuman primates involves long-term viral persistence in macrophages. J Clin Invest. 2010;120(3):894-906. 